\title{
RANDOM FIXED POINT THEOREMS FOR GENERALIZED RANDOM $\alpha-\psi$-CONTRACTIVE MAPPINGS WITH APPLICATIONS TO STOCHASTIC DIFFERENTIAL EQUATION
}

\author{
CHAYUT KONGBAN ${ }^{1}$, POOM KUMAM ${ }^{1,2, \S}$, JUAN MARTÍNEZ-MORENO $^{3}$
}

${ }^{1}$ KMUTTFixed Point Research Laboratory, Department of Mathematics, Room SCL 802 Fixed Point Laboratory, Science Laboratory Building, Faculty of Science, King Mongkut's University of Technology Thonburi (KMUTT), 126 Pracha-Uthit Road, Bang Mod, Thrung Khru, Bangkok 10140, Thailand

${ }^{2}$ KMUTT-Fixed Point Theory and Applications Research Group (KMUTT-FPTA), Theoretical and Computational Science Center (TaCS), Science Laboratory Building, Faculty of Science, King Mongkut's University of Technology Thonburi (KMUTT),

126 Pracha-Uthit Road, Bang Mod, Thrung Khru, Bangkok 10140, Thailand

${ }^{3}$ Department of Mathematics, Faculty of Experimental Science, University of Jaén, Campus Las Lagunillas,s/n, 23071 Jaén, Spain

Email addresses: chayut_kb@hotmail.com (C. Kongban), poom.kum@kmutt.ac.th (P. Kumam), jmmoreno@ujaen.es (J. Martínez-Moreno)

ABSTRACT. In this paper, we prove some random fixed point theorems for generalized random $\alpha-\psi$-contractive mappings in a Polish space and, as some applications, we show the existence of random solutions of second order random differential equation.

Keywords : random fixed point, random $\alpha$-admissible with respect to $\eta$, generalized random $\alpha-$ $\psi$-contractive mapping.

Mathematics Subject Classification: $47 \mathrm{H} 10 ; 47 \mathrm{H} 40$.

\section{INTRODUCTION}

Random fixed point theorems are stochastic generalization of a classical fixed point theorems. Random fixed point theorems for contraction mapping in aPolish space, i.e., a separable complete metric space, were proved by Špaček [22], Hanš [5, 6]. Some random fixed point theorems play amain role in developing theory of random differential and random integral equations (see, $[2,8,15]$ ). In 1996, Mukhejea [16] proved the random fixed point theorem of Schauder's type in otomic probability measure space. In 1984, Sehgal and Waters [20] proved the random fixed point theorem of the classical Rothe's fixed point theorem. The random fixed point theory and applications developed very rapidly (see, Bharucha-Reid [3], Itoh [7], Beg and Shahzad [1], Li [14], Kumam et al. [10-13], Nieto [17]).

In 2012, Samet et al. [19] introduced a new concept of $\alpha-\psi$-contractive type and $\alpha$-admissible mappings and establish fixed point theorems for such mappings in complete metric spaces. Afterwards Karapinar and Samet [9] introduced the concepts of

\footnotetext{
$\S$ Corresponding author: poom.kum@kmutt.ac.th (P. Kumam).
} 
generalized $\alpha-\psi$-contractive type mapping. In 2013, Salimi et al. [18] modified the notion of $\alpha$-admissible and $\alpha-\psi$-contractive mappings and established certain fixed point theorems. Our results are proper generalizations of the recent results in $[9,19]$.

Rencently, Tchier and Vetro [21] introduced the concepts of random $\alpha$-admissible and random $\alpha-\psi$-contractive mappings and established random fixed point theorems.

The purpose of this paper is to prove some random fixed point theorems for generalized random $\alpha-\psi$-contractive mappings in a Polish space and, by using our main results, we show the existence of random solutions of second order random differential equation.

\section{PRELIMINARIES}

We denote the Borel $\sigma$-algebra on a metric space $M$ by $B(M)$. Let $(\Omega, \Sigma)$ be a measurable space with $\Sigma$ a $\sigma$-algebra of subsets of $\Omega$. So that by $\Sigma \times B(M)$ we mean the smallest $\sigma$-algebra on $\Omega \times M$ containing all the sets $A \times B$ (with $A \in \Sigma$ and $B \in B(M))$.

Definition 2.1. Let $(\Omega, \Sigma)$ be a measurable space, $M$ and $N$ be two metric spaces. A mapping $f: \Omega \times M \rightarrow N$ is called Carathéodory if, for all $m \in M$, the mapping $\omega \rightarrow f(\omega, m)$ is $(\Sigma, B(N))$-measurable $(\Sigma$-measurable for short) and, for all $\omega \in \Omega$, the mapping $m \rightarrow f(\omega, m)$ is continuous.

Theorem 2.2. [4] If $(\Omega, \Sigma)$ is a measurable space, $M$ is a separable metric space, $N$ is a metric space, and $f: \Omega \times M \rightarrow N$ is a Carathéodory mapping, then $f$ is $\Sigma \times B(M)-$ measurable.

Corollary 2.3. [4] If $(\Omega, \Sigma)$ is a measurable space, $M$ is a separable metric space, $N$ is a metric space, and $f: \Omega \times M \rightarrow N$ is a Carathéodory mapping, and $u: \Omega \rightarrow M$ is $\Sigma$-measurable, then mapping $\omega \rightarrow f(\omega, u(\omega))$ is a $\Sigma$-measurable mapping from $\Omega$ into $N$.

Definition 2.4. [4] Let $(\Omega, \Sigma)$ be a measurable space, $M$ a separable metric space and $N$ a metric space. A function $f: \Omega \times M \rightarrow N$ is said to be superpositionally measurable ( sup-measurable for short), if for all $u: \Omega \rightarrow M$ is $\Sigma$-measurable, the function $\omega \rightarrow f(\omega, u(\omega))$ is $\Sigma$-measurable from $\Omega$ into $N$.

Remark 2.5. [4] Corollary 2.3 says that a Carathéodory function is sup-measurable. Also, every $\Sigma \times B(M)$-measurable functions $f: \Omega \times M \rightarrow N$ is sup-measurable.

Definition 2.6. A mapping $f: \Omega \times M \rightarrow M$ ys called random operator whenever, for any $x \in M, f(\cdot, x)$ is $\Sigma$-measurable. So, a random fixed point of $f$ is $\Sigma$-measurable mapping $z: \Omega \times M$ such that $z(\omega)=f(\omega, z(\omega))$ for all $\omega \in \Omega$.

Lemma 2.7. Let $M, N$ be two locally compact metric spaces. A mapping $f: \Omega \times M \rightarrow N$ is Carathéodory if and only if the mapping $\omega \rightarrow r(\omega)(\cdot)=f(\omega, \cdot)$ is $\Sigma$-measurable from $\Omega$ to $C(M, N)$ (i.e., the space of all continuous functions from $M$ into $N$ endowed with the compact-open topology).

Let $\Psi$ be the family of all nondecreasing functions $\psi:[0,+\infty) \rightarrow[0,+\infty)$ such that $\sum_{n=1}^{+\infty} \psi^{n}(t)<+\infty$ for each $t>0$, where $\psi^{n}$ denote the $n$th iterate of $\psi$.

Lemma 2.8. For every nondecreasing function $\psi:[0,+\infty) \rightarrow[0,+\infty)$, the following implication holds:

$$
\forall t>0, \quad \lim _{n \rightarrow+\infty} \psi^{n}(t)=0 \Longrightarrow \psi(t)<t
$$


Definition 2.9. Let $T: \Omega \times M \rightarrow M$ and $\alpha: \Omega \times M \times M \rightarrow[0,+\infty)$. We say that $T$ is a random $\alpha$-admissible if

$$
u, v \in M, \omega \in \Omega, \quad \alpha(\omega, u, v) \geq 1 \Longrightarrow \alpha(\omega, T(\omega, u), T(\omega, v)) \geq 1 .
$$

Definition 2.10. Let $(\Omega, \Sigma)$ be a measurable space, $(M, d)$ be a separable metric space, and $T: \Omega \times M \rightarrow M$ be a given mapping. We say that $T$ is a random $\alpha-\psi-$ contractive mapping if there exist functions $\alpha: \Omega \times M \times M \rightarrow[0,+\infty)$ and $\psi_{\omega} \in \Psi, \omega \in \Omega$, such that

$$
\alpha(\omega, u, v) d(T(\omega, u), T(\omega, v)) \leq \psi_{\omega}(d(u, v))
$$

for all $u, v \in M$ and $\omega \in \Omega$ such that $\alpha(\omega, u, v) \geq 1$.

\section{Main Results}

Definition 3.1. Let $T: \Omega \times M \rightarrow M$ and $\alpha, \eta: \Omega \times M \times M \rightarrow[0,+\infty)$. We say that $T$ is a random $\alpha$-admissible with respect to $\eta$ if

$u, v \in M, \omega \in \Omega, \quad \alpha(\omega, u, v) \geq \eta(\omega, u, v) \Rightarrow \alpha(\omega, T(\omega, u), T(\omega, v)) \geq \eta(\omega, T(\omega, u), T(\omega, v))$.

Note that if we take $\eta(\omega, u, v)=1$, then this definition reduces to Definition 2.9.

Definition 3.2. Let $(\Omega, \Sigma)$ be a measurable space, $(M, d)$ be a separable space, and $T: \Omega \times M \rightarrow M$ be a given mapping. We say that $T$ is a generalized random $\alpha-$ $\psi$-contractive mapping if there exist functions $\alpha, \eta: \Omega \times M \times M \rightarrow[0,+\infty)$ and $\psi_{\omega} \in \Psi, \omega \in \Omega$, such that

$$
\alpha(\omega, u, v) \geq \eta(\omega, u, v) \Rightarrow d(T(\omega, u), T(\omega, v)) \leq \psi_{\omega}(O(\omega,(u, v))),
$$

where

$O(\omega,(u, v))=\max \left\{d(u, v), \frac{d(u, T(\omega, u))+d(v, T(\omega, v))}{2}, \frac{d(u, T(\omega, v))+d(v, T(\omega, u))}{2}\right\}$ for all $u, v \in M$ and $\omega \in \Omega$.

Theorem 3.3. Let $(\Omega, \Sigma)$ be a measurable space, $(M, d)$ be a Polish space, $T: \Omega \times M \rightarrow$ $M$ and $\alpha, \eta: \Omega \times M \times M \rightarrow[0,+\infty)$. The hypotheses are the following:

(H1) $T$ is a random $\alpha$-admissible with respect to $\eta$.

(H2) there exists a measurable mapping $u_{0}: \Omega \rightarrow M$ such that, for all $\omega \in \Omega$.

$$
\alpha\left(\omega, u_{0}(\omega), T\left(\omega, u_{0}(\omega)\right)\right) \geq \eta\left(\omega, u_{0}(\omega), T\left(\omega, u_{0}(\omega)\right)\right) .
$$

(H3) $T$ is a Carathéodory mapping.

(H4) $T$ is a generalized random $\alpha-\psi$-contractive mapping.

Then $T$ has a random fixed point, that is, there exists $\zeta: \Omega \rightarrow M$ is measurable such that $T(\omega, \zeta(\omega))=\zeta(\omega)$ for all $\omega \in \Omega$.

Proof. Hypothese (H2) ensures that there exists a measurable mapping $u_{0}: \Omega \rightarrow M$ such that

$$
\alpha\left(\omega, u_{0}(\omega), T\left(\omega, u_{0}(\omega)\right)\right) \geq \eta\left(\omega, u_{0}(\omega), T\left(\omega, u_{0}(\omega)\right)\right),
$$

for all $\omega \in \Omega$. Define the sequence $\left\{u_{n}(\omega)\right\}$ in $M$ by

$$
u_{n}(\omega)=T^{n}\left(\omega, u_{0}(\omega)\right)=T\left(\omega, u_{n-1}(\omega)\right) \text { for all } n \in \mathbb{N} \cup\{0\}, \omega \in \Omega .
$$

If $u_{n}(\omega)=u_{n+1}(\omega)$ for all $n \in \mathbb{N} \cup\{0\}$, for all $\omega \in \Omega$, then $\zeta(\omega)=u_{n}(\omega)$ is a random fixed point of $T$. 
Assume that $u_{n}(\omega) \neq u_{n+1}(\omega)$ for all $n \in \mathbb{N} \cup\{0\}$, for one $\omega \in \Omega$. Since $T$ is a random $\alpha$-admissible with respect to $\eta(\mathrm{H} 1)$ and $\alpha\left(\omega, u_{0}(\omega), T\left(\omega, u_{0}(\omega)\right)\right)=\eta\left(\omega, u_{0}(\omega), T\left(\omega, u_{0}(\omega)\right)\right)$ we have

$$
\begin{aligned}
& \alpha\left(\omega, u_{1}(\omega), u_{2}(\omega)\right)=\alpha\left(\omega, T\left(\omega, u_{0}(\omega)\right), T^{2}\left(\omega, u_{0}(\omega)\right)\right) \\
& \geq \eta\left(\omega, T\left(\omega, u_{0}(\omega)\right), T^{2}\left(\omega, u_{0}(\omega)\right)\right)=\eta\left(\omega, u_{1}(\omega), u_{2}(\omega)\right) .
\end{aligned}
$$

Continuing this process, we get

$$
\alpha\left(\omega, u_{n}(\omega), u_{n+1}(\omega)\right) \geq \eta\left(\omega, u_{n}(\omega), u_{n+1}(\omega)\right) \text { for all } n \in \mathbb{N} \cup\{0\}, \omega \in \Omega \text {. }
$$

So, by (3.2) and hypothesis (H4) with $u=u_{n-1}(\omega), v=u_{n}(\omega)$, we get

$$
d\left(T\left(\omega, u_{n-1}(\omega)\right), T\left(\omega, u_{n}(\omega)\right)\right) \leq \psi_{\omega}\left(O\left(\omega,\left(u_{n-1}(\omega), u_{n}(\omega)\right)\right)\right) .
$$

On the other hand,

$$
\begin{aligned}
O\left(\omega,\left(u_{n-1}(\omega), u_{n}(\omega)\right)\right)= & \max \left\{d\left(u_{n-1}(\omega), u_{n}(\omega)\right),\right. \\
& \frac{d\left(u_{n-1}(\omega), T\left(\omega, u_{n-1}(\omega)\right)\right)+d\left(u_{n}(\omega), T\left(\omega, u_{n}(\omega)\right)\right)}{2}, \\
& \left.\frac{d\left(u_{n-1}(\omega), T\left(\omega, u_{n}(\omega)\right)\right)+d\left(u_{n}(\omega), T\left(\omega, u_{n-1}(\omega)\right)\right)}{2}\right\} \\
= & \max \left\{d\left(u_{n-1}(\omega), u_{n}(\omega)\right),\right. \\
& \frac{d\left(u_{n-1}(\omega), u_{n}(\omega)\right)+d\left(u_{n}(\omega), u_{n+1}(\omega)\right)}{2}, \\
\leq & \max \left\{d\left(u_{n-1}(\omega), u_{n}(\omega)\right),\right. \\
& \left.\frac{d\left(u_{n-1}(\omega), u_{n}(\omega)\right)+d\left(u_{n}(\omega), u_{n+1}(\omega)\right)}{2}\right\} \\
\leq & \max \left\{d\left(u_{n-1}(\omega), u_{n}(\omega)\right), d\left(u_{n}(\omega), u_{n+1}(\omega)\right)\right\}
\end{aligned}
$$

which implies

$$
d\left(u_{n}(\omega), u_{n+1}(\omega)\right) \leq \psi_{\omega}\left(\max \left\{d\left(u_{n-1}(\omega), u_{n}(\omega)\right), d\left(u_{n}(\omega), u_{n+1}(\omega)\right)\right\}\right) .
$$

Now, if $\max \left\{d\left(u_{n-1}(\omega), u_{n}(\omega)\right), d\left(u_{n}(\omega), u_{n+1}(\omega)\right)\right\}=d\left(u_{n}(\omega), u_{n+1}(\omega)\right)$ for all $n \in \mathbb{N}$, then

$$
\begin{aligned}
d\left(u_{n}(\omega), u_{n+1}(\omega)\right) & \leq \psi_{\omega}\left(\max \left\{d\left(u_{n-1}(\omega), u_{n}(\omega)\right), d\left(u_{n}(\omega), u_{n+1}(\omega)\right)\right\}\right) \\
& =\psi_{\omega}\left(d\left(u_{n}(\omega), u_{n+1}(\omega)\right)\right) \\
& <d\left(u_{n}(\omega), u_{n+1}(\omega)\right),
\end{aligned}
$$

which is a contradiction. Hence, for all $n \in \mathbb{N}$, we have

$$
d\left(u_{n}(\omega), u_{n+1}(\omega)\right) \leq \psi_{\omega} d\left(u_{n-1}(\omega), u_{n}(\omega)\right) .
$$

By induction, we have

$$
d\left(u_{n}(\omega), u_{n+1}(\omega)\right) \leq \psi_{\omega}^{n} d\left(u_{0}(\omega), u_{1}(\omega)\right) .
$$


GENERALIZED RANDOM $\alpha-\psi$-CONTRACTIVE MAPPINGS

Fix $\epsilon>0$, and let $N \in \mathbb{N}$ such that

$$
\sum_{n \geq N} \psi_{\omega} d\left(u_{n}(\omega), u_{n+1}(\omega)\right)<\epsilon \text { for all } n \in \mathbb{N} .
$$

Also, let $n, m \in \mathbb{N}$ with $m>n \geq N$. Then, by the triangular inequality, we get

$$
\begin{aligned}
d\left(u_{n}(\omega), u_{m}(\omega)\right) & \leq \sum_{k=n}^{m-1} d\left(u_{k}(\omega), u_{k+1}(\omega)\right) \\
& \leq \sum_{k=n}^{m-1} \psi_{\omega}^{k}\left(d\left(u_{0}(\omega), u_{1}(\omega)\right)\right) \\
& \leq \sum_{n \geq n(\epsilon)} \psi_{\omega}^{n}\left(d\left(u_{0}(\omega), u_{1}(\omega)\right)\right) \\
& <\epsilon .
\end{aligned}
$$

The argument show that the sequence $\left\{u_{n}(\omega)\right\}$ is a Cauchy sequence. Since $(M, d)$ is complete, there exists $\zeta: \Omega \rightarrow M$ such that $u_{n}(\omega) \rightarrow \zeta(\omega)$ as $n \rightarrow+\infty$ for all $\omega \in \Omega$. Since $T$ is a Carathéodory mapping (hypothesis(H3)), it follows that $u_{n}$ is measurable for all $n \in \mathbb{N}$ and that $u_{n+1}(\omega)=T\left(\omega, u_{n}(\omega)\right) \rightarrow T(\omega, \zeta(\omega))$ as $n \rightarrow+\infty$ for all $\omega \in \Omega$. By the uniqueness of the limit, we get $\zeta(\omega)=T(\omega, \zeta(\omega))$, that is, $\zeta(\omega)$ is a random fixed point of $T$. Note that $\zeta$ is a measurable since it is a limit of a sequence of measurable.

By taking $\eta(\omega, u, v)=1, \forall \omega \in \Omega, u, v \in M$ in Theorem 3.3, we have the following result.

Corollary 3.4. Let $(\Omega, \Sigma)$ be a measurable space, $(M, d)$ be a Polish space, $T: \Omega \times M \rightarrow$ $M$ and $\alpha: \Omega \times M \times M \rightarrow[0,+\infty)$. The hypotheses are the following:

(H1) $T$ is a random $\alpha$-admissible.

(H2) there exists a measurable mapping $u_{0}: \Omega \rightarrow M$ such that, for all $\omega \in \Omega$.

$$
\alpha\left(\omega, u_{0}(\omega), T\left(\omega, u_{0}(\omega)\right)\right) \geq 1 \text {. }
$$

(H3) $T$ is a Carathéodory mapping.

(H4) $T$ is a generalized random $\alpha-\psi$-contractive mapping.

Then $T$ has a random fixed point, that is, there exists $\zeta: \Omega \rightarrow M$ is measurable such that $T(\omega, \zeta(\omega))=\zeta(\omega)$ for all $\omega \in \Omega$.

Theorem 3.5. Let $(\Omega, \Sigma)$ be a measurable space, $(M, d)$ be a Polish space, $T: \Omega \times M \rightarrow$ $M$ and $\alpha: \Omega \times M \times M \rightarrow[0,+\infty)$. The hypotheses are the following:

(G1) $T$ is a random $\alpha$-admissible with respect to $\eta$.

(G2) there exists a measurable mapping $u_{0}: \Omega \rightarrow M$ such that, for all $\omega \in \Omega$.

$$
\alpha\left(\omega, u_{0}(\omega), T\left(\omega, u_{0}(\omega)\right)\right) \geq \eta\left(\omega, u_{0}(\omega), T\left(\omega, u_{0}(\omega)\right)\right) .
$$

(G3) $T$ is a sup-measurable.

(G4) $T$ is a generalized random $\alpha-\psi$-contractive mapping.

(G5) If $\left\{u_{n}(\omega)\right\}$ is a sequence in $M$ such that

$$
\alpha\left(\omega, u_{n}(\omega), u_{n+1}(\omega)\right) \geq \eta\left(\omega, u_{n}(\omega), u_{n+1}(\omega)\right)
$$

for all $\omega \in \Omega$, for all $n \in \mathbb{N} \cup\{0\}$ and $u_{n}(\omega) \rightarrow u(\omega)$ as $n \rightarrow+\infty$, then

$$
\alpha\left(\omega, u_{n}(\omega), u(\omega)\right) \geq \eta\left(\omega, u_{n}(\omega), u(\omega)\right)
$$

for all $\omega \in \Omega$, for all $n \in \mathbb{N} \cup\{0\}$. 
Then $T$ has a random fixed point, that is, there exists $\zeta: \Omega \rightarrow M$ is measurable such that $T(\omega, \zeta(\omega))=\zeta(\omega)$ for all $\omega \in \Omega$.

Proof. A similar reasoning as in the proof of Theorem 3.3 gives us that the sequence $\left\{u_{n}(\omega)\right\}$ is a Cauchy sequence for all $\omega \in \Omega$. This means that there exists $\zeta: \Omega \rightarrow M$ such that $u_{n}(\omega) \rightarrow \zeta(\omega)$ as $n \rightarrow+\infty$ for all $\omega \in \Omega$. On the other hand, from (3.2) and hypothesis (G5), we have

$$
\alpha\left(\omega, u_{n}(\omega), \zeta(\omega)\right) \geq \eta\left(\omega, u_{n}(\omega), \zeta(\omega)\right) \text { for all } n \in \mathbb{N} \cup\{0\}, \omega \in \Omega .
$$

Now, using the triangle inequality (3.3) and (G4), we get

$$
\begin{aligned}
d(T(\omega, \zeta(\omega)), \zeta(\omega)) & \leq d\left(T(\omega, \zeta(\omega)), T\left(\omega, u_{n}(\omega)\right)\right)+d\left(u_{n+1}(\omega), \zeta(\omega)\right) \\
& \leq \psi_{\omega}\left(d\left(\zeta(\omega), u_{n}(\omega)\right)\right)+d\left(u_{n+1}(\omega), \zeta(\omega)\right) .
\end{aligned}
$$

Taking the limit as $n \rightarrow+\infty$ and since $\psi_{\omega}$ is continuous at $t=0$, we have

$$
d(T(\omega, \zeta(\omega)), \zeta(\omega))=0
$$

that is, $T(\omega, \zeta(\omega))=\zeta(\omega)$ for all $\omega \in \Omega$. The hypothesis that $T$ is sup-measurable implies that $u_{n}$ is measurable for all $n \in \mathbb{N}$ and hence $\zeta$ is measurable. Thus $\zeta$ is a random fixed point of $T$.

\section{Application to ordinary RANDOM Differential EQUations}

We consider the following two-point boundary value problem of second order random differential equation:

$$
\left\{\begin{array}{l}
-\frac{d^{2} u}{d t^{2}}(\omega, t)=f(\omega, t, u(\omega, t)), \quad t \in[0,1] \\
u(\omega, u)=u(\omega, 1)=0
\end{array}\right.
$$

for all $\omega \in \Omega$, we have $f: \Omega \times[0,1] \times \mathbb{R} \rightarrow \mathbb{R}$ has certain regularities and $\Omega$ is nonempty.

By a random solution of system (4.1), we mean a measurable mapping $u: \Omega \rightarrow$ $C([0,1], \mathbb{R})$ satisfying $(4.1)$, where $C([0,1], \mathbb{R})$ denote the space of all continuous functions defined on $[0,1]$. The space $C([0,1], \mathbb{R})$ endowed with the metric

$$
d_{\infty}(x, y)=\|x-y\|_{\infty} .
$$

In this section, we prove a theorem producing the existence of random soution of system (4.1).

Let $(\Omega, \Sigma)$ be a measurable space. Let $f: \Omega \times[0,1] \times \mathbb{R} \rightarrow \mathbb{R}$ be a Carathéodory function, which means that $\omega \mapsto f(\omega, t, u)$ is measurable for all $(t, u) \in[0,1] \times \mathbb{R}$ and $(t, u) \mapsto f(\omega, t, u)$ is continuous for all $\omega \in \Omega$.

Then consider the integral operator $F: \Omega \times C([0,1], \mathbb{R}) \rightarrow C([0,1], \mathbb{R})$ defined by

$$
F(\omega, u)(t)=\int_{0}^{1} G(t, s) f(\omega, s, u(s)) d s,
$$

for all $u \in C([0,1], \mathbb{R})$ and $\omega \in \Omega$, where $G: \mathbb{R} \times \mathbb{R} \rightarrow \mathbb{R}$ is continuous function, and $g: \Omega \times[0,1] \times \mathbb{R} \rightarrow \mathbb{R}$ is a Carathéodory function.

Remark 4.1. $F$ is a random operator from $\Omega \times C([0,1], \mathbb{R})$ into $C([0,1], \mathbb{R}$. In fact, given $u \in C([0,1], \mathbb{R})$ since $f$ is a Carathéodory function for $s \in[0,1]$ fixed, the function $h: \Omega \times[0,1] \rightarrow \mathbb{R}$, defined by $h(\omega, t)=G(t, s) f(\omega, s, u(s))$, is Carathéodory. By Lemma 2.7 , the integral in (4.2) is limit of a finite sum of measurable functions. So, the mapping $\omega \rightarrow F(\omega, u)$ is measurable, and hence $F$ is a random operator. 
GENERALIZED RANDOM $\alpha-\psi$-CONTRACTIVE MAPPINGS

Remark 4.2. Let $h: \Omega \times[0,1] \times \mathbb{R} \rightarrow \mathbb{R}$ be a Carathéodory function, $u \in C([0,1], \mathbb{R})$, and let $\left\{u_{n}\right\} \subset C([0,1], \mathbb{R})$ be a sequence convergent to $u$. Then there exists an interval $[a, b] \subset \mathbb{R}$ such that $u_{n}(s), u(s) \in[a, b]$ for all $s \in[0,1]$. The continuity of the function $h(\omega, \cdot, \cdot)$ in $[0,1] \times \mathbb{R}$ for fixed $\omega \in \Omega$ ensures that the function $h(\omega, \cdot, \cdot)$ is uniformly continuous in $[0,1] \times[a, b]$.

The hypotheses are the following:

(i) For each $\omega \in \Omega$, there exist $\psi_{\omega} \in \Psi$ and $\theta: \Omega \times \mathbb{R} \times \mathbb{R} \rightarrow \mathbb{R}$ such that if $\theta(\omega, a, b) \geq 0$ for all $a, b \in \mathbb{R}$, then for every $t \in[0,1]$, we have

$$
\begin{aligned}
& |f(\omega, t, a)-f(\omega, t, b)| \\
& \leq \psi_{\omega}\left(\operatorname { m a x } \left\{|a(t)-b(t)|, \frac{1}{2}[|a(t)-F(\omega, a(t))|+|b(t)-F(\omega, b(t))|],\right.\right. \\
& \left.\left.\frac{1}{2}[|a(t)-F(\omega, b(t))|+|b(t)-F(\omega, a(t))|]\right\}\right) .
\end{aligned}
$$

(ii) There exists a measurable mapping $u_{0}: \Omega \rightarrow C([0,1], \mathbb{R})$ such that, for all $\omega \in \Omega$, we have

$$
\theta\left(\omega, u_{0}(\omega)(t), F\left(\omega, u_{0}(\omega)\right)(t)\right) \geq 0 \quad \text { for all } t \in[0,1]
$$

(iii) For each $\omega \in \Omega$ and for all $t \in[0,1], u, v \in C([0,1], \mathbb{R})$, we have

$$
\theta(\omega, u(t), v(t)) \geq 0 \Rightarrow \theta(\omega, F(\omega, u)(t), F(\omega, v)(t)) \geq 0 .
$$

(iv) $\int_{0}^{1} G(t, s) d s \leq 1$ for all $t \in[0,1]$ and $s \in[0,1]$.

Theorem 4.3. If hypotheses $(i)-(i v)$ hold, then the random integral operator $F$ has a random fixed point.

Proof. For fixed $\omega \in \Omega$ we show that $F(\omega, \cdot)$ is continuous. Indeed, consider a sequence $\left\{u_{n}\right\} \in C([0,1], \mathbb{R})$ with $u_{n} \rightarrow u \in C([0,1], \mathbb{R})$ as $n \rightarrow+\infty$. By Remark 4.2, there exists $[a, b] \subset \mathbb{R}$ such that $u_{n}(s), u(s) \in[a, b]$ for all $s \in[0,1]$. In addition, the functions $f(\omega, \cdot, \cdot)$ is uniformly continuous in $[0,1] \times[a, b]$. Thus, for fixed $\epsilon>0$, there exists $\delta>0$ such that

$$
\left|f\left(\omega, s_{1}, u_{1}\right)-f\left(\omega, s_{2}, u_{2}\right)\right|<\epsilon,
$$

for all $s_{1}, s_{2} \in[0,1]$ and $u_{1}, u_{2} \in[a, b]$ such that $\left|s_{1}-s_{2}\right|+\left|u_{1}-u_{2}\right|<\delta$.

Now, let $n(\delta) \in \mathbb{N}$ such that $\left\|u_{n}-u\right\|_{\infty}<\delta$ whenever $n \geq n(\delta)$. Then, for every $n \geq n(\delta)$, we have

$$
\left|f\left(\omega, s, u_{n}(s)\right)-f(\omega, s, u(s))\right|<\epsilon .
$$

Consequently, for $t \in[0,1]$ and $n \geq n(\delta)$, we have

$$
\begin{aligned}
&\left|F\left(\omega, u_{n}\right)(t)-F(\omega, u)(t)\right| \leq \int_{0}^{1}\left|G(t, s) \| f\left(\omega, s, u_{n}(s)\right)-f(\omega, s, u(s))\right| d s \\
& \leq \epsilon \\
& \Rightarrow\left\|F\left(\omega, u_{n}\right)-F(\omega, u)\right\|_{\infty} \leq \epsilon .
\end{aligned}
$$

So, $d_{\infty}\left(F\left(\omega, u_{n}\right), F(\omega, u)\right) \rightarrow 0$ as $n \rightarrow+\infty \Rightarrow F(\omega, \cdot)$ is a continuous operator for each fixed $\omega \in \Omega$.

Thus, by Remark 4.2, $F: \Omega \times C([0,1], \mathbb{R}) \rightarrow C([0,1], \mathbb{R})$ is a Carathéodory function. 
Next step is to show that the integral operator $F$ satisfies a generalized random $\alpha-\psi$-contractive type condition as in (H4). So, for each $\omega \in \Omega$ and all $u, v \in C([0,1], \mathbb{R})$ such that $\theta(\omega, u(t), v(t)) \geq 0$ for all $t \in[0,1]$, we prove that

$$
d_{\infty}(F(\omega, u), F(\omega, v)) \leq \psi_{\omega}(O(\omega,(u, v)))
$$

where

$O(\omega,(u, v))=\max \left\{d(u, v), \frac{d(u, F(\omega, u))+d(v, F(\omega, v))}{2}, \frac{d(u, F(\omega, v))+d(v, F(\omega, u))}{2}\right\}$.

Indeed, let $\omega \in \Omega$ be fixed, and $u, v \in C([0,1], \mathbb{R})$ be such that $\theta(\omega, u(t), v(t)) \geq 0$ for all $t \in[0,1]$, then

$$
\begin{aligned}
&|F(\omega, u)(t)-F(\omega, v)(t)| \\
&=\left|\int_{0}^{1} G(t, s)[f(\omega, s, u(s))-f(\omega, s, v(s))] d s\right| \\
& \leq \int_{0}^{1} G(t, s)|f(\omega, s, u(s))-f(\omega, s, v(s))| d s \\
& \leq \int_{0}^{1} G(t, s)\left[\psi _ { \omega } \left(\operatorname { m a x } \left\{|u(s)-v(s)|, \frac{1}{2}[|u(s)-F(\omega, u(s))|+|v(s)-F(\omega, v(s))|],\right.\right.\right. \\
&\left.\left.\left.\frac{1}{2}[|u(s)-F(\omega, v(s))|+|v(s)-F(\omega, u(s))|]\right\}\right)\right] d s \\
& \leq \int_{0}^{1} G(t, s)\left[\psi _ { \omega } \left(\operatorname { m a x } \left\{\mid u(s)-v(s) \|, \frac{1}{2}[\|u(s)-F(\omega, u(s))\|+\|v(s)-F(\omega, v(s))\|],\right.\right.\right. \\
&\left.\left.\left.\frac{1}{2}[\|u(s)-F(\omega, v(s))\|+\|v(s)-F(\omega, u(s))\|]\right\}\right)\right] d s \\
&=\left(\int_{0}^{1} G(t, s) d s\right) \psi_{\omega}\left(\operatorname { m a x } \left\{\mid u(s)-v(s) \|, \frac{1}{2}[\|u(s)-F(\omega, u(s))\|+\|v(s)-F(\omega, v(s))\|],\right.\right. \\
&\left.\left.\frac{1}{2}[\|u(s)-F(\omega, v(s))\|+\|v(s)-F(\omega, u(s))\|]\right\}\right) \\
& \leq \psi_{\omega}\left(\operatorname { m a x } \left\{\mid u(s)-v(s) \|, \frac{1}{2}[\|u(s)-F(\omega, u(s))\|+\|v(s)-F(\omega, v(s))\|],\right.\right. \\
&\left.\left.\frac{1}{2}[\|u(s)-F(\omega, v(s))\|+\|v(s)-F(\omega, u(s))\|]\right\}\right) .
\end{aligned}
$$

Then

$$
\begin{aligned}
& \|F(\omega, u)-F(\omega, v)\| \\
& \leq \psi_{\omega}\left(\operatorname { m a x } \left\{\mid u(s)-v(s) \|, \frac{1}{2}[\|u(s)-F(\omega, u(s))\|+\|v(s)-F(\omega, v(s))\|],\right.\right. \\
& \left.\left.\frac{1}{2}[\|u(s)-F(\omega, v(s))\|+\|v(s)-F(\omega, u(s))\|]\right\}\right) .
\end{aligned}
$$

Let $\alpha: \Omega \times C([0,1], \mathbb{R}) \times C([0,1], \mathbb{R}) \rightarrow[0,+\infty)$ be function given as

$$
\alpha(\omega, u, v)= \begin{cases}1 & \text { if } \theta(\omega, u(t), v(t)) \geq 0 \quad \text { for all } t \in[0,1] \\ 0 & \text { otherwise }\end{cases}
$$


for all $\omega \in \Omega$. So, for all $u, v \in C([0,1], \mathbb{R})$ with $\alpha(\omega, u, v) \geq 1$, we get

$$
\begin{aligned}
& \|F(\omega, u)-F(\omega, v)\|_{\infty} \\
& \leq \psi_{\omega}\left(\operatorname { m a x } \left\{\mid u(s)-v(s) \|_{\infty}, \frac{1}{2}\left[\|u(s)-F(\omega, u(s))\|_{\infty}+\|v(s)-F(\omega, v(s))\|_{\infty}\right],\right.\right. \\
& \left.\left.\frac{1}{2}\left[\|u(s)-F(\omega, v(s))\|_{\infty}+\|v(s)-F(\omega, u(s))\|_{\infty}\right]\right\}\right),
\end{aligned}
$$

which means that $F$ is a generalized random $\alpha-\psi$-contractive integral operator.

Note thar, for each $\omega \in \Omega$ and all $t \in[0,1], u, v \in C([0,1], \mathbb{R})$, we have

$$
\begin{aligned}
& \alpha(\omega, u, v) \geq 1 \\
& \Rightarrow \theta(\omega, u(t), v(t)) \geq 0 \quad \text { for all } t \in[0,1] \\
& \Rightarrow \theta(\omega, F(\omega, u)(t), F(\omega, v)(t)) \geq 0 \\
& \alpha(\omega, F(\omega, u), F(\omega, v)) \geq 1,
\end{aligned}
$$

which means that $F$ is a random $\alpha$-admissible integral oprator.

All of the hypotheses of Corollary 3.4 are satisfied, and hence the mapping $F$ has a random fixed point.

\section{ACKNOWLEDGMENTS}

The first author thanks for the support of Petchra Pra Jom Klao Doctoral Scholarship for Ph.D. program of King Mongkut's University of Technology Thonburi (KMUTT). This work was completed while the first author visit Prof. Juan Martínez-Moreno at University of Jaén, Jaén, Spain. The authors thank very much Prof. Juan MartínezMoreno for his hospitality and support.

\section{REFERENCES}

[1] I. Beg and N. Shahzad. Random fixed points of random multivalued operators on Polish spaces. Nonlinear Anal., 20(7):835-847, 1993.

[2] A. T. Bharucha-Reid. Random integral equations. Academic Press, New York-London, 1972. Mathematics in Science and Engineering, Vol. 96.

[3] A. T. Bharucha-Reid. Fixed point theorems in probabilistic analysis. Bull. Amer. Math. Soc., 82(5):641-657, 1976.

[4] Z. Denkowski, S. Migorski, and N. S. Papageorgiou. An introduction to nonlinear analysis: theory. Kluwer Academic Publishers, Boston, MA, 2003.

[5] O. Hanš. Reduzierende zufällige Transformationen. Czechoslovak Math. J., 7(82):154-158, 1957.

[6] O. Hanš. Random operator equations. In Proc. 4th Berkeley Sympos. Math. Statist. and Prob., Vol. II, pages 185-202. Univ. California Press, Berkeley, Calif., 1961.

[7] S. Itoh. A random fixed point theorem for a multivalued contraction mapping. Pacific J. Math., 68(1):85-90, 1977.

[8] S. Itoh. Random fixed-point theorems with an application to random differential equations in Banach spaces. J. Math. Anal. Appl., 67(2):261-273, 1979.

[9] E. Karapinar and B. Samet. Generalized $\alpha-\psi$ contractive type mappings and related fixed point theorems with applications. Abstr. Appl. Anal., pages Art. ID 793486, 17, 2012.

[10] P. Kumam. Random common fixed points of single-valued and multivalued random operators in a uniformly convex Banach space. J. Comput. Anal. Appl., 13(2):368-375, 2011.

[11] P. Kumam and S. Plubtieng. Some random fixed point theorems for random asymptotically regular operators. Demonstratio Math., 42(1):131-141, 2009.

[12] W. Kumam and P. Kumam. Random fixed points of multivalued random operators with property (D). Random Oper. Stoch. Equ., 15(2):127-136, 2007. 
[13] W. Kumam and P. Kumam. Random fixed point theorems for multivalued subsequentially limitcontractive maps satisfying inwardness conditions. J. Comput. Anal. Appl., 14(2):239-251, 2012.

[14] G. Li and H. Duan. On random fixed point theorems of random monotone operators. Appl. Math. Lett., 18(9):1019-1026, 2005.

[15] T.-C. Lin. Random approximations and random fixed point theorems for non-self-maps. Proc. Amer. Math. Soc., 103(4):1129-1135, 1988.

[16] A. Mukherjea. Random transformations on banach spaces. ProQuest LLC, Ann Arbor, MI, 1967. Thesis (Ph.D.)-Wayne State University.

[17] J. J. Nieto, A. Ouahab, and R. Rodriguez-López. Random fixed point theorems in partially ordered metric spaces. Fixed Point Theory Appl., pages Paper No. 98, 19, 2016.

[18] P. Salimi, A. Latif, and N. Hussain. Modified $\alpha$ - $\psi$-contractive mappings with applications. Fixed Point Theory Appl., pages 2013:151, 19, 2013.

[19] B. Samet, C. Vetro, and P. Vetro. Fixed point theorems for $\alpha-\psi$-contractive type mappings. Nonlinear Anal., 75(4):2154-2165, 2012.

[20] V. M. Sehgal and C. Waters. Some random fixed point theorems for condensing operators. Proc. Amer. Math. Soc., 90(3):425-429, 1984.

[21] F. Tchier and C. Vetro. Some notes on a second-order random boundary value problem. Nonlinear Anal. Model. Control, 22(6):808-820, 2017.

[22] A. Špaček. Zufällige gleichungen. Czechoslovak Mathematical Journal, 05(4):462-466, 1955. 